January 2021

"A political economy of loose means-testing in targeted social programs"

Helmuth Cremer, Justina Klimaviciute and Pierre Pestieau 


\title{
A political economy of loose means-testing in targeted social $\operatorname{programs}^{1}$
}

\author{
Helmuth Cremer ${ }^{2}$, Justina Klimaviciute ${ }^{3}$ and Pierre Pestieau ${ }^{4}$
}

January 2021

\footnotetext{
${ }^{1}$ Financial support from the Chaire "Marché des risques et creation de valeur" of the FdR/SCOR is gratefully acknowledged. Helmuth Cremer gratefully acknowledges the funding received by TSE from ANR under grant ANR-17-EURE-0010 (Investissements d'Avenir program).

${ }^{2}$ Corresponding author, Toulouse School of Economics, University of Toulouse Capitole, France. E-mail: helmuth.cremer@tse-fr.eu

${ }^{3}$ Faculty of Economics and Business Administration, Vilnius University, Lithuania. E-mail: justina.klimaviciute@evaf.vu.lt

${ }^{4}$ University of Liege and CORE, Université Catholique de Louvain, Belgium and Toulouse School of Economics, France. E-mail: p.pestieau@ulg.ac.be
} 


\begin{abstract}
This paper studies the political sustainability of programs that are targeted towards the poor. Given that the poor to whom these programs cater do not constitute a majority, we show that for their own good it pays to let the middle class benefit from them in a random way. This approach mimics the actual institutional arrangements whereby middle-class individuals feel that they can successfully apply to the programs. We consider a two stage decision process: first a Rawlsian government chooses the probability at which the middle class is allowed to benefit from a given program; then, majority voting determines the level of benefit and the rate of contribution. At the first, constitutional stage, the government cannot commit to a specific level of taxes and benefit but anticipates that these are set by majority voting in the second stage.
\end{abstract}

Keywords: Targeted transfers; Political support; Redistribution paradox.

JEL classification: H23; D72; H50 


\section{Introduction}

In a number of countries there exist means-testing assistance targeted towards the poor. They cover all sorts of needs: health, housing, pensions, income maintenance, long-term care (LTC). In this paper, we use as an example LTC assistance programs. These programs are supposed to cover those who cannot purchase private insurance or self-insure. One, however, observes that in most cases there are loopholes in meanstesting: individuals who could afford paying for their LTC end up receiving benefits from the program. In other words, means-testing is not always strictly enforced and this has ramifications, which have hitherto received little attention. For example, in many US states, an elderly person may own a home valued at $\$ 802,000$, plus home furnishings, jewellery and an automobile of uncapped value while receiving long-term Medicaid support. In addition, they are allowed to have various life insurance policies, retirement accounts with unlimited assets and a defined-benefit pension plan. By most standards, such a person is considered wealthy and yet he benefits from an assistance program. ${ }^{1}$ We would like to understand why governments allow for such exceptions in their means-testing policy. Our conjecture is that these exceptions are designed in such a way that the LTC program gets enough political support. The idea is that to have a majority in favor of a LTC assistance program one needs to add to the population of poor part of the middle class. This issue is related to the literature on the redistribution paradox ${ }^{2}$ and that on the political economy of social security $^{3}$. The message of this line of research is that the only way to ensure the political sustainability of a public scheme is to allow the middle class to benefit in part from it. Alternatively, the idea is that social benefits should be partially related to either earnings or contributions. The gist of all that literature is that "a program for the poor is a poor program".

De Donder and Peluso (2018) also show that public aid received by a minority of the population may be supported by majority voting when voters see the attribution of the aid as being stochastic. However, their analysis is merely of a positive nature, and they do not consider policy design which is our main focus. We first show that for the good of the poorest part of the population it pays to let the middle class benefit from the public scheme in a random way that mimics the actual institutional arrangements whereby middle-class individuals feel that they can successfully apply to the program. We then explore the optimal choice of the government in more detail. The model we have in mind comprises two stages. In the first stage the government decides about the probability that the middle class can benefit from the public scheme, with the objective of maximizing the welfare of the worst-off, and in the second stage there is a majority vote on the level of benefits. We consider a society made of three types of individuals: the poor, the middle-class and the rich. The poor benefit from the public scheme with probability one, the middle class with a probability to be determined and the rich with a zero probability. The government levies a tax of rate $\tau$ to finance a flat public benefit $g$.

The paper is organized as follows. Section 2 presents the model, while section 3 discusses the second

\footnotetext{
${ }^{1}$ Cremer and Pestieau (2018)

${ }^{2}$ Korpi and Palme (1998)

${ }^{3}$ Casamatta et al. $(2000 \mathrm{a}, \mathrm{b})$
} 
stage of the game, i.e. the problem of a middle-class individual who is decisive in the majority vote. Section 4 analyzes the first stage, i.e. the problem of the government, by first studying the analytical problem and then discussing some numerical results. Finally, the last section provides some concluding remarks.

\section{Model}

Consider a society with 3 types of individuals: poor (type 1), middle-class (type 2) and rich (type 3 ), with exogenous incomes $w_{1}<w_{2}<w_{3}$. Their shares in the society $n_{1}, n_{2}$ and $n_{3}$ are such that $n_{1}<1 / 2$, $n_{1}+n_{2}>1 / 2$ and $n_{1}+n_{2}+n_{3}=1$. In words, the poor and the middle class represent a majority, but no group includes more than half of total population.

We study a public scheme which provides a means-tested benefit $g$ financed by a linear income tax at rate $\tau$. We consider a single period in which the three types of individuals may benefit from the scheme with different probabilities. Let $\pi_{i} \in[0,1]$ (for $i=1,2,3$ ) denote the probability for type $i$ to qualify for the means-tested benefit $g$. Expected utility of a type $i$ individual is given by:

$$
U_{i}=\pi_{i} u\left(w_{i}(1-\tau)+g\right)+\left(1-\pi_{i}\right) u\left(w_{i}(1-\tau)\right)
$$

with $u^{\prime}(\cdot)>0$ and $u^{\prime \prime}(\cdot)<0 .^{4}$

We further assume that $\pi_{1}=1$ and $\pi_{3}=0$, while $\pi_{2}$ is to be determined. In other words, we assume that the poor benefit from the public scheme for sure, the rich can never benefit from it, whereas it may be optimal for the government to leave some loopholes in the means-testing so that the middle class has a non-zero chance to benefit from the program.

The model has two stages. In the first one, the government chooses the level of $\pi_{2}$ by maximizing the Rawlsian social welfare function, that is the welfare of the worst-off (type 1) individuals. In the second stage, there is a majority vote on the level of benefits or, equivalently, on the level of the tax rate $\tau$. First period decisions are made anticipating the induced voting equilibrium in stage 2 .

Proceeding by backward induction, we start by solving the second stage. From our assumptions it is clear that the median voter is a type 2 individual. Therefore, given the government's chosen $\pi_{2}$, the resulting tax rate will be the one preferred by the individuals of type 2 . We analyze the problem of a type 2 individual in the next section.

\footnotetext{
${ }^{4}$ In the case of LTC, this can be seen as focusing on elderly individuals in need of LTC. In other words, we can assume that all individuals in the model need LTC, but not everyone qualifies for the public aid. Moreover, we can focus on the individuals' post-retirement stage, i.e. instead of modeling explicitly their consumption and saving decisions made before retirement, assume that the individuals save a constant share $s$ of their disposable income and consume the rest. We can then abstract from the individuals' utility of consumption before retirement and normalize $s$ to 1 , which amounts to saying that the individuals arrive to the post-retirement stage with a wealth equal to $w_{i}(1-\tau)$, for $i=1,2,3$.
} 


\section{Problem of a middle-class individual}

Given the government's chosen $\pi_{2}$, the utility of a middle-class (type 2) individual writes as

$$
U_{2}=\pi_{2} u\left(w_{2}(1-\tau)+g\right)+\left(1-\pi_{2}\right) u\left(w_{2}(1-\tau)\right)
$$

The levels of $\tau$ and $g$ are related by the government's budget constraint which is given by

$$
\left(\pi_{2} n_{2}+n_{1}\right) g=\tau \bar{w}
$$

where $\bar{w}=w_{1} n_{1}+w_{2} n_{2}+w_{3} n_{3}$ is the average income. From this it follows that

$$
g=\frac{\tau \bar{w}}{\left(\pi_{2} n_{2}+n_{1}\right)} \quad \text { and } \quad \frac{\partial g}{\partial \tau}=\frac{\bar{w}}{\left(\pi_{2} n_{2}+n_{1}\right)}>0
$$

The level of $\tau$ preferred by a middle-class individual is determined by the following first-order condition (FOC):

$$
\frac{\partial U_{2}}{\partial \tau}=\pi_{2} u^{\prime}\left(c_{2}\right)\left[-w_{2}+\frac{\bar{w}}{\left(\pi_{2} n_{2}+n_{1}\right)}\right]-\left(1-\pi_{2}\right) u^{\prime}\left(\tilde{c}_{2}\right) w_{2} \leq 0,
$$

where $c_{2}=w_{2}(1-\tau)+g$ and $\tilde{c}_{2}=w_{2}(1-\tau)$.

The expression shows that a higher tax rate has two effects on the individual. On the one hand, it creates a cost by reducing the individual's disposable income; on the other hand, it creates a benefit by increasing the public transfer that the individual may receive if he is "lucky". The cost is certain, while the benefit is obtained with probability $\pi_{2}$.

We first derive the condition for $\tau>0$. To do this, we set $\tau=0$, which implies $g=0$ and so $u^{\prime}\left(c_{2}\right)=u^{\prime}\left(\tilde{c}_{2}\right)$. Using this in (1), the condition for $\tau>0$ is

$$
\begin{gathered}
u^{\prime}\left(c_{2}\right)\left[-w_{2}+\pi_{2} \frac{\bar{w}}{\left(\pi_{2} n_{2}+n_{1}\right)}\right]>0 \\
\Leftrightarrow \\
\pi_{2}>\frac{w_{2} n_{1}}{\left(\bar{w}-w_{2} n_{2}\right)}=\hat{\pi}_{2}
\end{gathered}
$$

This expression shows that type 2 individuals vote for a strictly positive tax rate, and thus a non-zero public benefit when their probability to benefit from the public scheme is high enough, and clearly strictly positive. In other words, the public scheme can be politically sustainable only when the probability for the middle class to receive the benefits is sufficiently large. ${ }^{5}$

\footnotetext{
${ }^{5}$ We assume $w_{2} n_{1}<\bar{w}-w_{2} n_{2}$ because otherwise, $w_{2} n_{1} /\left(\bar{w}-w_{2} n_{2}\right) \geq 1$, and so $\pi_{2}$ would have to be greater than 1 .
} 
Assuming that this condition holds, so that there is an interior solution for $\tau$, we have

$$
\frac{\partial U_{2}}{\partial \tau}=\pi_{2} u^{\prime}\left(c_{2}\right)\left[-w_{2}+\frac{\bar{w}}{\left(\pi_{2} n_{2}+n_{1}\right)}\right]-\left(1-\pi_{2}\right) u^{\prime}\left(\tilde{c}_{2}\right) w_{2}=0
$$

Differentiating this expression yields:

$$
\frac{\partial \tau}{\partial \pi_{2}}=\frac{u^{\prime}\left(c_{2}\right) \frac{\bar{w} n_{1}}{\left(\pi_{2} n_{2}+n_{1}\right)^{2}}-\pi_{2} u^{\prime \prime}\left(c_{2}\right) \frac{\tau \bar{w} n_{2}}{\left(\pi_{2} n_{2}+n_{1}\right)^{2}}\left[-w_{2}+\frac{\bar{w}}{\left(\pi_{2} n_{2}+n_{1}\right)}\right]+w_{2}\left[u^{\prime}\left(\tilde{c}_{2}\right)-u^{\prime}\left(c_{2}\right)\right]}{-S O C_{\tau}}>0
$$

where

$$
S O C_{\tau}=\pi_{2} u^{\prime \prime}\left(c_{2}\right)\left[-w_{2}+\frac{\bar{w}}{\left(\pi_{2} n_{2}+n_{1}\right)}\right]^{2}+\left(1-\pi_{2}\right) u^{\prime \prime}\left(\tilde{c}_{2}\right) w_{2}^{2}<0
$$

is the second-order condition for $\tau$, which is satisfied given the concavity of $u$. Consequently, the voting equilibrium tax rate increases with $\pi_{2}$.

\section{Problem of the government}

We now move to the first stage where the government has to determine the optimal level of $\pi_{2}$. We first derive the problem analytically and then provide some numerical results.

\subsection{Analytical problem}

We consider a Rawlsian government which maximizes the utility of the worst-off (type 1) individuals. The problem of the government is given by

$$
\max _{\pi_{2}} U_{1}=u\left(w_{1}(1-\tau)+\frac{\tau \bar{w}}{\left(\pi_{2} n_{2}+n_{1}\right)}\right)
$$

which yields the following FOC:

$$
\frac{\partial U_{1}}{\partial \pi_{2}}=u^{\prime}\left(c_{1}\right)\left[\frac{\frac{\partial \tau}{\partial \pi_{2}}\left(\pi_{2} n_{2}+n_{1}\right)\left[\bar{w}-w_{1}\left(\pi_{2} n_{2}+n_{1}\right)\right]-\tau \bar{w} n_{2}}{\left(\pi_{2} n_{2}+n_{1}\right)^{2}}\right] \leq 0
$$

where

$$
c_{1}=w_{1}(1-\tau)+\frac{\tau \bar{w}}{\left(\pi_{2} n_{2}+n_{1}\right)} .
$$

First, we can easily see that having $\pi_{2} \leq \hat{\pi}_{2}$ is not optimal. Recall that $\hat{\pi}_{2}$, defined by (2) is the threshold which has to be exceeded to obtain a strictly positive voting equilibrium tax rate. Consequently, 
for $\pi_{2} \leq \hat{\pi}_{2}$, the majority vote would result in $\tau=0$ and it can be easily verified that

$$
\left.\frac{\partial \tau}{\partial \pi_{2}}\right|_{\pi_{2}=\hat{\pi}_{2}}>0
$$

which gives

$$
\left.\frac{\partial U_{1}}{\partial \pi_{2}}\right|_{\pi_{2}=\hat{\pi}_{2}}=u^{\prime}\left(w_{1}\right)\left[\frac{\left.\frac{\partial \tau}{\partial \pi_{2}}\right|_{\pi_{2}=\hat{\pi}_{2}}\left[\bar{w}-w_{1}\left(\hat{\pi}_{2} n_{2}+n_{1}\right)\right]}{\left(\hat{\pi}_{2} n_{2}+n_{1}\right)}\right]>0
$$

Consequently, even though the government is concerned only about the well-being of the individuals of type 1 , it is still optimal to allow the type 2 individuals to benefit from the public scheme with a non-zero probability.

Focusing on an interior solution for $\pi_{2}$, we have

$$
\frac{\partial U_{1}}{\partial \pi_{2}}=u^{\prime}\left(c_{1}\right)\left[\frac{\frac{\partial \tau}{\partial \pi_{2}}\left(\pi_{2} n_{2}+n_{1}\right)\left[\bar{w}-w_{1}\left(\pi_{2} n_{2}+n_{1}\right)\right]-\tau \bar{w} n_{2}}{\left(\pi_{2} n_{2}+n_{1}\right)^{2}}\right]=0
$$

This expression shows that the choice of $\pi_{2}$ involves a tradeoff. On the one hand, making it easier for the middle class to benefit from the scheme increases the number of the beneficiaries of the program and thus, for a given tax revenue, decreases the size of the benefit obtained by each recipient. This is reflected by the second term in the numerator of (4). On the other hand, a higher $\pi_{2}$ ensures a higher equilibrium tax rate, which has two effects. First, it allows increasing the tax revenue and thus the level of the public benefit. However, it also means that the poor will have to pay higher taxes, which is a negative effect on them. Nevertheless, because the first term in the numerator of (4) can easily be shown to be positive, the positive impact of the increase in the public benefit outweighs the negative effect of higher taxes for the poor. To sum up, a higher equilibrium tax rate overall affects the individuals of type 1 positively. Therefore, the optimal level of $\pi_{2}$ needs to strike a balance between this positive effect of being able to sustain a higher tax rate and the negative effect of having to divide the public benefit among a larger number of individuals.

We further investigate this problem by considering a specific utility function and looking at some numerical examples.

\subsection{Numerical results}

We assume that the individuals' utility function is iso-elastic, i.e.

$$
u(x)=\frac{x^{1-\alpha}}{1-\alpha},
$$


with $\alpha<1$. With this utility function, from (3) we have

$$
\tau=\frac{\left(1-\pi_{2}\right)^{-\frac{1}{\alpha}} w_{2}^{\left(1-\frac{1}{\alpha}\right)}-\pi_{2}^{-\frac{1}{\alpha}}\left[-w_{2}+\frac{\bar{w}}{\left(\pi_{2} n_{2}+n_{1}\right)}\right]^{-\frac{1}{\alpha}} w_{2}}{\left(1-\pi_{2}\right)^{-\frac{1}{\alpha}} w_{2}^{\left(1-\frac{1}{\alpha}\right)}+\pi_{2}^{-\frac{1}{\alpha}}\left[-w_{2}+\frac{\bar{w}}{\left(\pi_{2} n_{2}+n_{1}\right)}\right]^{\left(1-\frac{1}{\alpha}\right)}}
$$

We provide some numerical examples in which we explore how the optimal value of $\pi_{2}$ and the resulting level of $\tau$ are affected by variations in the model's parameters.

\section{Example 1: changes in the average income $\bar{w}$}

We fix $\alpha=1 / 3, w_{1}=0.4, w_{2}=1, n_{2}=0.9, n_{1}=0.05$ and vary the income of the rich $w_{3}$ to generate different levels of the average income $\bar{w}$. We follow the usual assumption that the average income is higher than the median income $\left(w_{2}\right)$. Table 1 presents the optimal values of $\pi_{2}$ and the resulting values of $\tau$ for different levels of $\bar{w}$.

Table 1: Changes in $\bar{w}$

\begin{tabular}{|c|c|c||c|c|c||c|c|c|}
\hline $\bar{w}$ & $\pi_{2}$ & $\tau$ & $\bar{w}$ & $\pi_{2}$ & $\tau$ & $\bar{w}$ & $\pi_{2}$ & $\tau$ \\
\hline 1.1 & 0.912359 & 0.939071 & 1.6 & 0.403743 & 0.62108 & 2.1 & 0.264809 & 0.592558 \\
\hline 1.2 & 0.778954 & 0.839325 & 1.7 & 0.362559 & 0.608064 & 2.2 & 0.249406 & 0.592772 \\
\hline 1.3 & 0.638997 & 0.74196 & 1.8 & 0.331594 & 0.602422 & 2.3 & 0.236076 & 0.593735 \\
\hline 1.4 & 0.53309 & 0.679252 & 1.9 & 0.304295 & 0.595725 & 2.4 & 0.224409 & 0.59524 \\
\hline 1.5 & 0.45819 & 0.642688 & 2 & 0.282844 & 0.593394 & 2.5 & 0.214099 & 0.597141 \\
\hline
\end{tabular}

As we can see from the table, the optimal value of $\pi_{2}$ decreases when $\bar{w}$ goes up, but it seems that the decreases are sharper at lower values of $\bar{w}$ than at higher ones. On the other hand, the relationship between the equilibrium tax rate and $\bar{w}$ is non monotonic: at first, $\tau$ is decreasing with $\bar{w}$, but when $\bar{w}$ reaches a sufficiently high level, $\tau$ starts increasing with further increases in $\bar{w}$. This can be understood by noting that $\tau$ depends on $\bar{w}$ in two ways: directly and through $\pi_{2}$. It can be verified from (5) that the direct effect is positive (indeed, when the average income is higher, there is more to gain from increasing the tax rate), while we also know that $\partial \tau / \partial \pi_{2}>0$. At lower levels of $\bar{w}$, there are strong decreases in $\pi_{2}$, which results in $\tau$ decreasing as well, but at higher levels of $\bar{w}$, when the decreases in $\pi_{2}$ are not that strong, the positive direct effect of $\bar{w}$ starts prevailing and so $\tau$ starts increasing. Note, however, that this happens only when the income inequality, that is the difference between $\bar{w}$ and $w_{2}$, is substantially large.

\section{Example 2: changes in the median income $w_{2}$}

We fix $\alpha=1 / 3, w_{1}=0.4, \bar{w}=2, n_{2}=0.9, n_{1}=0.05$ and vary the level of the median income $w_{2}$. Since we are keeping $\bar{w}$ fixed as $w_{2}$ changes we are also varying $w_{3}$ (but in the opposite direction). Again 
we assume that the average income is higher than the median income. The optimal values of $\pi_{2}$ and the resulting values of $\tau$ for different levels of $w_{2}$ are presented in Table 2.

Table 2: Changes in $w_{2}$

\begin{tabular}{|c|c|c||c|c|c|}
\hline$w_{2}$ & $\pi_{2}$ & $\tau$ & $w_{2}$ & $\pi_{2}$ & $\tau$ \\
\hline 0.6 & 0.157256 & 0.613959 & 1.3 & 0.447337 & 0.650371 \\
\hline 0.7 & 0.183455 & 0.601075 & 1.4 & 0.530541 & 0.69581 \\
\hline 0.8 & 0.212429 & 0.593275 & 1.5 & 0.632935 & 0.760523 \\
\hline 0.9 & 0.245135 & 0.590556 & 1.6 & 0.746212 & 0.838318 \\
\hline 1 & 0.282844 & 0.593394 & 1.7 & 0.847216 & 0.908221 \\
\hline 1.1 & 0.327308 & 0.602853 & 1.8 & 0.919531 & 0.955396 \\
\hline 1.2 & 0.380995 & 0.620824 & 1.9 & 0.964649 & 0.98207 \\
\hline
\end{tabular}

We can see from the table that the optimal value of $\pi_{2}$ increases when $w_{2}$ goes up. The increases are smaller at lower values of $w_{2}$, then become stronger as $w_{2}$ increases and tend to become a bit smaller again at high levels of $w_{2}$. The reaction of $\tau$ is again non monotonic: at lower levels of $w_{2}$ it is decreasing, but then starts going up, even though the rise slows down at high levels of $w_{2}$. The underlying intuition is again that $\tau$ depends on $w_{2}$ both directly and through $\pi_{2}$. It can be verified from (5) that the direct effect is negative: indeed, a higher $w_{2}$ increases the cost of taxation to the individuals of type 2 . On the other hand, increases in $\pi_{2}$ affect the equilibrium tax rate positively. At lower levels of $w_{2}$, when the increases in $\pi_{2}$ are small, the negative direct effect prevails and $\tau$ is decreasing. However, when the increases in $\pi_{2}$ become larger, $\tau$ starts increasing because of the positive indirect effect. As the rise in $\pi_{2}$ slows down at high levels of $w_{2}$, so does the rise in $\tau$, even though the increasing trend remains.

\section{Example 3: changes in $\alpha$}

Finally, we explore the impact of the parameter $\alpha$ of the iso-elastic utility function which represents the relative risk aversion of the individual. We fix $w_{1}=0.4, w_{2}=1, \bar{w}=2, n_{2}=0.9, n_{1}=0.05$ and vary $\alpha$. The optimal values of $\pi_{2}$ and the resulting values of $\tau$ for different levels of $\alpha$ are presented in Table 4 .

Table 3: Changes in $\alpha$

\begin{tabular}{|c|c|c||c|c|c|}
\hline$\alpha$ & $\pi_{2}$ & $\tau$ & $\alpha$ & $\pi_{2}$ & $\tau$ \\
\hline $1 / 5$ & 0.184006 & 0.699958 & $1 / 2$ & 0.436216 & 0.586621 \\
\hline $1 / 4$ & 0.220198 & 0.647212 & $2 / 3$ & 0.684658 & 0.728688 \\
\hline $1 / 3$ & 0.282844 & 0.593394 & $3 / 4$ & 0.839802 & 0.852863 \\
\hline
\end{tabular}

It can be seen from the table that the optimal value of $\pi_{2}$ is increasing in $\alpha$. Intuitively, when the middle-class individuals become more risk averse, a higher probability for them to benefit from the public scheme is needed to ensure their support for the program. On the other hand, the effect of $\alpha$ on $\tau$ is 
non monotonic: the tax rate first decreases and then starts increasing with $\alpha$. This can be explained by the following reasoning. Intuitively, a more risk averse individual prefers a lower tax rate, and this effect prevails at lower levels of $\alpha$. However, at higher levels of $\alpha$, this negative effect becomes outweighed by the positive impact of a rapidly increasing probability to benefit from the public scheme, which reverses the direction of the change in $\tau$.

\section{Conclusion}

There is a long-standing controversy over the question of whether targeting social transfers towards the bottom part of the income distribution actually enhances or weakens their redistributive impact. From an efficiency standpoint, targeting is clearly desirable, but at the same time it lacks political sustainability. One indeed observes an inverse relationship at the country level between social transfer targeting and redistributive impact. As observed by Korpi and Palme (1998), "the more we target benefits at the poor, the less likely we are to reduce poverty and inequality". In this paper we address this question through a simple political economy model that reflects the idea that the middle class would be allowed to benefit from social transfers in a stochastic way. In the real world, there are many factors that can explain why some people have access to benefits to which they are not primarily entitled and others do not. They are related to risk aversion, compliance level, public spirit, etc. Studying these factors is beyond the scope of our paper. Here we limit ourselves to show that there may exist a (strictly positive) probability of providing the social transfers to the middle class that strikes a balance between social optimality and political sustainability.

To assess how this probability and the resulting tax evolve when the parameters of the model change, we had to resort to a numerical illustration. The main finding of this simulation is that as income inequality increases (measured by the difference between average income and the income of the middle class) the probability of letting the middle-class benefit from the public program decreases consistently; see exmaples 1 and 2. In other words, means testing should be enforced in a stricter way in unequal societies than in more egalitarian countries. Intuitively, as $w_{3}$ increases, type 2 benefits more from the program so that a Rawlsian government can decrease $\pi_{2}$ which leaves more resources for the poor, while continuing to ensure political support. Another interesting finding concerns risk aversion. As the coefficient of relative risk aversion increases, the probability of the middle-class benefiting from the public program increases all the way. Since benefits are random for the middle class, it takes a higher probability is required to "bribe" them into supporting the program. These results are interesting for their own sake because they provide guidance for the design of meants-tested public policies and show how these are affected by two of a society's main characteristics, namely inequality and risk aversion. Since these characteristics are country specific the results also provide testable implications that can be explored in empirical studies. 


\section{References}

[1] Casamatta, G., Cremer, H. and Pestieau, P. (2000a) "The political economy of social security", Scandinavian Journal of Economics, 102, 502-522.

[2] Casamatta, G., Cremer, H. and Pestieau, P. (2000b) "Political sustainability and the design of social insurance", Journal of Public Economics, 75, 315-340.

[3] Cremer, H. and Pestieau, P. (2018) "Means-Tested Long-Term Care and Family Transfers", German Economic Review, 19(3), 351-364.

[4] De Donder, P. and Peluso, E. (2018) "Politically sustainable targeted transfers", Public Choice, 174, 301-313.

[5] Korpi, W. and Palme, J. (1998) "The paradox of redistribution and strategies of equality: Welfare state institutions, inequality and poverty in the Western countries", American Sociological Review, 63, 661-687. 